\title{
INVESTIGANDO O PROCESSO DE ESCOLHA: UMA ANÁLISE DAS ESTRATÉGIAS DE DECISÃO UTILIZADAS NA COMPRA DE TELEVISORES LCD
}

\author{
Mauri Leodir Löbler \\ mllobler@gmail.com \\ Universidade Federal de Santa Maria - Santa Maria, RS / Brasil \\ Eliete dos Reis \\ elietedosreis@gmail.com \\ Universidade Federal de Santa Maria - Santa Maria, RS / Brasil \\ Larissa Medianeira Bolzan \\ larissambolzan@gmail.com \\ Universidade Federal de Santa Maria - Santa Maria, RS / Brasil
}

\begin{abstract}
Recebido em 16/06/2012
Aprovado em 04/07/2013

Disponibilizado em 01/04/2014

Avaliado pelo sistema double blind review

Revista Eletrônica de Administração

Editor: Luís Felipe Nascimento

ISSN 1413-2311 (versão on-line)

Editada pela Escola de Administração da Universidade Federal do Rio Grande do Sul.

Periodicidade: Quadrimestral

Sistema requerido: Adobe Acrobat Reader.
\end{abstract}

\section{RESUMO}

Tendo-se em vista a importância de entender como os indivíduos fazem suas escolhas e utilizam diferentes estratégias para tomar decisões, frente a uma diversidade de alternativas, este estudo tem como objetivo verificar quais variáveis explicam a utilização de estratégias compensatórias e não compensatórias nas decisões de compra dos consumidores. As estratégias compensatórias permitem a utilização de trade-offs entre um valor alto em uma dimensão e um valor baixo em outra (KIM; CHUNG; PARADICE, 1997; KAHN, 2005), enquanto nas estratégias não compensatórias valores baixos não podem ser compensados (WESTENBERG; KOELE, 1994). Este trabalho descreve um estudo de casos múltiplos, de caráter qualitativo e de natureza exploratória, utilizando-se como objeto de análise televisores Liquid Crystal Display (LCD). Como instrumento de verificação das estratégias de decisão do consumidor, foram realizadas dez entrevistas em profundidade, cujo roteiro foi elaborado com base em manuais técnicos, com questões abertas, que permitiam ao entrevistado contribuir com o que julgasse relevante. A análise dos dados permitiu concluir que o conhecimento prévio do produto é o que determina a utilização de diferentes estratégias de decisão. Neste estudo, os consumidores que antes da decisão final de compra buscaram informações a respeito do produto foram caracterizados como consumidores com conhecimento. Com relação ao ponto em que os indivíduos mudam o tipo de estratégias de decisão, os resultados da pesquisa revelaram que os consumidores, quando frente a dois ou três televisores LCD para a escolha final, iniciam os trade-offs, caracterizando, assim, a mudança de estratégias de decisão de não compensatória para compensatória. Salienta-se que os consumidores com pouco conhecimento fazem uso de estratégias não compensatórias em maior número do que 
aqueles com conhecimento, os quais se utilizam mais de estratégias compensatórias. Acrescenta-se também que os tomadores de decisão não se utilizaram dos mesmos atributos na etapa compensatória, uma vez que, nessa fase, os indivíduos com maior conhecimento a respeito do produto realizaram trade-offs com foco na performance das alternativas.

Palavras-chave: Decisão de compra; Estratégias de decisão; Estratégia compensatória; Estratégia não compensatória; Trade-offs.

\title{
INVESTIGATING THE CHOOSING PROCESS: AN ANALYSIS OF DECISION STRATEGIES USED TO BUY LCD TELEVISIONS
}

\begin{abstract}
Considering the importance of understanding how individuals make their choices and use different strategies to make decisions with a variety of alternatives, this study has aimed to determine which variables explain the use of compensatory and non-compensatory strategies in purchasing decisions made by the consumers. The compensatory strategies allow the use of trade-offs between a high value on an attribute and a low value in another (KIM, CHUNG; PARADICE, 1997; KAHN, 2005). Meanwhile, the non-compensatory strategies, low values cannot be compensated (WESTENBERG; KOELE, 1994). This study may be described as a multiple case study, also qualitative and exploratory, and the object of analysis used is Liquid Crystal Display (LCD) televisions. The data collection consisted of 10 deep interviews, the questions were developed based on technical manuals, and they were open questions, which allowed the respondent to contribute with what they judge relevant. The data analysis showed that previous knowledge of the product is what determines the use of different decision strategies. In this study, consumers who, before the final purchase decision, had sought information about the product were characterized as consumers with knowledge. Regarding the point where individuals have changed their strategies of decision, the results of the survey revealed that the consumers, when facing two or three LCD TVs, in the final decision moment, begin the trade-offs, characterizing the change of decision strategies from noncompensatory to compensatory. It is worth noting that consumers with little knowledge tend to use non-compensatory strategies more than those with more knowledge, who tend to use more compensatory strategies. Decision makers did not use the same attributes in the compensatory stage, since individuals with greater knowledge about the product made tradeoffs focusing on the performance of the alternatives.
\end{abstract}

Keywords: Buying decision; Decision strategies; Compensatory strategy; Non-compensatory strategy; Trade-offs.

\section{INVESTIGANDO EL PROCESO DE SELECCIÓN: UN ANÁLISIS DE LAS ESTRATEGIAS DE DECISIÓN UTILIZADOS EN LA COMPRA DE TELEVISORES LCD}

RESUMEN

REAd | Porto Alegre - Edição 77 - N 1 - janeiro/abril 2014 - p. 170-194 
Investigando o processo de escolha: uma análise das estratégias de decisão utilizadas na compra de televisores LCD

Dada la importancia de comprender cómo los individuos toman sus decisiones y utilizan diferentes estrategias para tomar decisiones, este estudio tiene como objetivo verificar que variables explican el uso de estrategias no compensatorias y estrategias compensatorias en las decisiones de compra de los consumidores. Las estrategias de compensación permiten el uso de las compensaciones entre un valor alto en un atributo y un valor bajo en otro (KIM; CHUNG; PARADICE, 1997; KAHN, 2005). En las estrategias compensatorias los valores bajos no pueden ser compensados (WESTENBERG; KOELE, 1994). Este artículo describe un estudio de casos múltiples, cualitativos y de carácter exploratorio, utilizando como objeto de análisis de televisores de Liquid Crystal Display (LCD). Como herramienta para colección de datos se realizaron 10 encuestas. Los análisis de los datos mostraron que el conocimiento previo del producto es lo que determina el uso de estrategias de decisión diferente. En este estudio, los consumidores que antes de la decisión final de compra, solicitan información sobre el producto caracterizan los consumidores con conocimiento. Los individuos cambian el tipo de estrategias de decisión, cuando se han afrontado con dos o tres televisores LCD, entonces ellos empiezan el trade-off, y es eso que caracteriza el cambio de las estrategias de decisión. En el paso no compensatorio del proceso de decisiones, se han eliminado todos los productos cuyos atributos no habían alcanzado el nivel aceptable. En el paso compensatorio, las personas compensan un menor valor en un atributo en lugar de un valor más alto en otra. Resalta que los consumidores con poco conocimiento hacen uso de las estrategias no compensatorias en mayor número de aquellos con conocimientos, los cuales se utilizan más estrategias compensatorias. También se agrega que los tomadores de decisiones no han utilizado los mismos atributos en el paso de compensación, ya que esta fase las personas con mayor conocimiento acerca del producto hecho compensaciones con un enfoque en el rendimiento de las alternativas.

Palabras Clave: Decisión de compra; Estrategias de decisión; La estrategia de compensación; La estrategia de no compensación; Trade-offs.

\section{INTRODUÇÃO}

O modo como as pessoas fazem escolhas foi de grande interesse para pesquisadores de vários campos que, ao longo do tempo, produziram muitos estudos e teorias sobre o comportamento humano de escolha (ENGEL; BLACKWELL; MINIARD, 2000). Nos últimos 20 anos, foram identificadas diferentes estratégias utilizadas pelos indivíduos para tomar decisões (PAYNE; BETTMAN; JOHNSON, 1993). Segundo Bettman e Zins (1977), o indivíduo pode realizar ou implementar o processo de escolha sob diferentes formas. Nesse contexto, de acordo com Bueno e Yamaguti (2009), a tomada de decisão humana pode transitar em termos de sua importância relativa, de modo que algumas características no processo decisório tornam-se semelhantes em situações distintas, como no momento da aquisição de um bem de consumo ou como diante de uma crucial escolha de negócios.

Dessa forma, têm-se como verdadeiras as considerações de Payne, Bettman e Johnson (1993), que afirmam ser a flexibilidade com que o indivíduo responde a uma ampla variedade de condições na solução de um problema um dos aspectos mais fascinantes do REAd | Porto Alegre - Edição 77 - N 1 - janeiro/abril 2014 - p. 170-194 
comportamento de decisão humana. A partir disso, pode-se concluir que o processo decisório humano é não linear, e essa não linearidade resulta na escolha efetiva (KIM; YANG; KIM, 2008).

Com relação às regras de decisão que representam as estratégias utilizadas pelos indivíduos para fazer uma seleção das alternativas de escolha, algumas se valem de todas as informações relevantes, envolvendo as alternativas disponíveis para simplificação (heurística); enquanto outras usam informações mais selecionadas e tendem a encerrar em trade-offs, ou trocas (ENGEL; BLACKWELL; MINIARD, 2000). Como consequência do uso de estratégias heurísticas, as pessoas, às vezes, cometem erros substanciais de decisão (PAYNE; BETTMAN; JOHNSON, 1993). Torna-se relevante mencionar que trade-offs ou trocas ocorrem quando um ponto fraco percebido em um atributo pode ser contrabalançado pelo ponto forte percebido em outro atributo, envolvendo, então, uma estratégia compensatória (ENGEL; BLACKWELL; MINIARD, 2000). As estratégias que tendem a evitar os trade-offs são classificadas como não compensatórias (decisão não linear) (KIM; CHUNG; PARADICE, 1997). De acordo com Engel, Blackwell e Miniard (2000), essas estratégias ou regras de decisão não compensatórias caracterizam-se pelo fato de o ponto fraco em um atributo não poder ser compensado por seu desempenho forte em outro atributo.

Nesse contexto, o estudo se substancia por possibilitar a compreensão de como os indivíduos tomam decisões frente a uma diversidade de alternativas. Assim, o objetivo geral deste estudo está centrado em analisar a forma como os indivíduos se utilizam de estratégias compensatórias e não compensatórias nas decisões de compra. Visando-se a atingir o objetivo proposto, foram definidos os seguintes objetivos específicos, que balizaram a realização desta pesquisa: (i) identificar características nos indivíduos pesquisados que possam diferenciar estratégias de decisão de compra; e (ii) verificar em que ponto os indivíduos mudam o tipo de estratégia de decisão.

A fim de situar o presente artigo num campo específico, processo decisório, foi utilizado como referência Simon, que, em 1959, classificou a área da Economia em quatro campos de estudo: trabalho, oligopólio e teorias da competição, processo decisório e teoria do desenvolvimento econômico. Economia, à época, predominava, pois ainda não haviam sido desenvolvidos campos mais específicos como Administração ou suas subáreas.

Processo decisório como disciplina surge por volta de 1960, segundo Connolly, Arkes e Hammond (2000), por meio do aumento de estudos empíricos sistemáticos. Esse fenômeno ocorre devido à intenção de os pesquisadores aumentarem o entendimento sobre 
Investigando o processo de escolha: uma análise das estratégias de decisão utilizadas na compra de televisores LCD

psicologia cognitiva, incluindo aí os estudos da memória, pensamentos, solução de problemas, imagem mental e linguagens.

Processo decisório é um campo específico de estudos dentro da área de Economia, e seus desdobramentos históricos, como a Administração, pertencem ao campo de estudos sobre comportamento humano. Normalmente, os trabalhos empíricos sobre processo decisório envolvem a tomada de decisão sobre decisões financeiras, decisões de compra de consumidores, mas os estudos, assim como o presente artigo, preocupam-se com o comportamento decisório embora se valendo de estudos destas outras áreas mais específicas, que serão referenciadas, dada a relevância das contribuições da área de Marketing (no campo do assim chamado "comportamento do consumidor") para a compreensão do fenômeno do processo de decisão em si.

Para atingir o objetivo proposto, o trabalho está estruturado em mais cinco seções, além da introdução: na seção dois, aborda-se o referencial teórico que balizou a realização deste estudo; na seção três, é apresentada a arquitetura metodológica que possibilitou a operacionalização da pesquisa; na seção quatro, são apresentados os resultados, alinhados com os objetivos propostos; na seção cinco, abordam-se as considerações finais, seguidas das referências bibliográficas utilizadas no estudo em questão.

\section{REFERENCIAL TEÓRICO}

\subsection{Tomada de decisão}

De acordo com Harrison (1996), uma decisão é definida como um momento num processo contínuo de avaliação de alternativas, para satisfazer um objetivo, em que as expectativas sobre determinado curso de ação estimulam o tomador de decisão a selecionar seu direcionamento mais provável ao cumprimento desse objetivo. Nessa mesma linha, para Markman e Medin (2001), situações de decisão são geralmente definidas como aquelas em que o tomador de decisão tem algum objetivo insatisfeito e um conjunto de opções para satisfazer o mesmo, tendo então que avaliar as opções e selecionar uma.

Dessa forma, pesquisas sobre decisão têm apresentado que o comportamento de decisão humana é amplamente suscetível a uma grande variedade de tarefas e de um contexto de fatores (PAYNE; BETTMAN; JOHNSON, 1993). De alguma maneira, os indivíduos organizam esses fatores e tomam decisões que são lógicas e correntes para eles (ENGEL; BLACKWELL; MINIARD, 2000). No entender de Payne, Bettman e Johnson (1993), um 
mesmo indivíduo, muitas vezes, utiliza diferentes processos de tomada de decisão, em razão de seus julgamentos, para escolher algumas alternativas em detrimento de outras ou para decidir entre um conjunto de boas opções versus um conjunto de más opções. Assim, a seleção de estratégias, em outras palavras, é altamente dependente das propriedades do problema de decisão (PAYNE; BETTMAN; JOHNSON, 1993).

Nesse sentido, para Tversky (1972), pessoas, quando confrontadas com uma escolha entre várias alternativas, frequentemente sofrem com a incerteza e apresentam inconsistência em suas decisões, ou seja, as pessoas, muitas vezes, não têm certeza de quais alternativas devem selecionar e nem sempre fazem a mesma escolha em condições aparentemente idênticas. As estratégias para o processamento da informação serão diferentes, dependendo do número de alternativas a considerar (PAYNE; BETTMAN; JOHNSON, 1993). Assim, segundo Kim, Yang e Kim (2008), pesquisas indicam que o processo de decisão humana é, muitas vezes, não linear, e modelos não lineares seriam mais adequados para o desenvolvimento de modelos avançados de tomada de decisão.

Apesar da evidência da não linearidade no processo de decisão humana, modelos conceituais de decisão têm surgido a fim de sintetizar os principais mecanismos que orientam a tomada de decisão e o processo decisório. Esses modelos sugerem que o esforço pode ser a chave para explicar o comportamento de decisão (TODD; BENBASAT, 1991).

Um dos modelos do processo decisório, desenvolvido por Shimizu (2001), consiste em duas fases: a formulação do problema e a tomada de decisão. Na primeira fase, o decisor percebe e define o problema (necessidade ou oportunidade) de decisão, determinando as variáveis (parâmetros) relevantes ao processo; após, ele estabelece os critérios ou objetivos da decisão, relacionando critérios com parâmetros, ou seja, modelando o problema; quando o problema está modelado, o decisor gera alternativas de decisão e prevê os possíveis cenários para os diferentes valores dos parâmetros. Na segunda, o decisor avalia as alternativas geradas na fase primeira e escolhe a que melhor satisfaz os objetivos; então, a decisão é tomada, e é realizado um monitoramento dos resultados.

Shimizu (2001) ainda afirma que um procedimento de extrema importância que antecede e acompanha as fases um e dois do modelo proposto é a coleta e análise dos dados para a tomada de decisão. Nesse sentido, Dieckmann, Dippold e Dietrich (2009) apresentam uma forma para coletar dados: o método do perfil completo. Perfis de produtos que abrangem todas as características relevantes, avaliados tanto pela classificação ou ranking quanto 
Investigando o processo de escolha: uma análise das estratégias de decisão utilizadas na compra de televisores LCD

escolha discreta. Trata-se de uma estratégia de decisão em que todas as informações disponíveis sobre o objeto de decisão são ponderadas.

Além dos modelos conceituais de decisão, é importante considerar que o comportamento do processo decisório pode variar, de acordo com Engel, Blackwell e Miniard (2000), conforme o grau em que as alternativas são diferenciadas, a presença ou ausência de restrição de tempo e o grau de envolvimento no contexto de necessidades e motivações importantes. Para Lesgold et al. (1988), o processo decisório pode variar de acordo com o nível de conhecimento. Nessa mesma linha de pensamento, Brooks, Leblanc e Norman (2000) afirmam que a decisão também depende da percepção da informação por parte do indivíduo, a qual dependerá, em parte, das informações armazenadas na memória do decisor, da experiência do consumidor com uma marca, por exemplo. O conhecimento a respeito do objeto de decisão é ativado durante o processo decisório.

\subsection{Estratégias de decisão}

Os indivíduos realizam suas decisões de modo distinto, e uma forma de distinção pode ser dada a partir de estratégias de decisão (LÖBLER, 2005). Segundo Engel, Blackwell e Miniard (2000), estratégias de decisão são procedimentos utilizados para realização da escolha final em um processo decisório. Estratégias de decisão são como uma sequência de operações mentais usados para transformar um estado inicial de conhecimento em um objetivo final do conhecimento, no qual o decisor vê o problema de decisão como particularmente resolvido (PAYNE; BETTMAN; JOHNSON, 1993). Na ótica de Westenberg e Koele (1994), as estratégias de decisão são avaliações do processo que conduzem a uma escolha, baseadas no modo como os indivíduos processam as informações disponíveis, considerando alternativas com múltiplos atributos, a fim de chegar a uma escolha final.

Nesse sentindo, as estratégias decisão podem variar de procedimentos bem simplistas, que demandam pouco esforço e tempo, até aqueles muito elaborados, que envolvem consideravelmente mais tempo e esforço de processamento por parte dos indivíduos (ENGEL; BLACKWELL; MINIARD, 2000). Nesta mesma linha, para Payne, Bettman e Johnson (1993), indivíduos utilizam múltiplas estratégias de decisão em diferentes situações, incluindo vários métodos de simplificação ou escolhas heurísticas, ou seja, uma resposta adaptativa à capacidade limitada do processamento da informação quanto às exigências das tarefas de decisões complexas. 
Segundo Payne, Bettman e Johnson (1993), muitas pesquisas sugerem que as estratégias utilizadas para o processamento da informação serão diferentes, dependendo do número de alternativas a considerar. Dessa forma, as pessoas, quando se deparam com problemas de decisão que envolvem exatamente duas ou três alternativas, frequentemente utilizam estratégias de decisão que processam todas as informações relevantes e determinam explicitamente uma para decidir em que medida estão propensos a fazer trade-off, ou trocas, de um atributo menos valorizado para outro atributo mais valorizado.

Por outro lado, pessoas, quando enfrentam problemas complexos de escolha, ou seja, envolvendo muitas alternativas, frequentemente adotam estratégias de simplificação (heurística) que são mais seletivas na utilização da informação (PAYNE; BETTMAN; JOHNSON, 1993). Conforme estudo de Löbler e Estivalete (2008), decisores novatos ou com pouco conhecimento a respeito do objeto de decisão necessitam de aliviar o processo mental devido à sua capacidade mais limitada de organizar informações acerca de um objeto e, dessa forma, utilizam-se mais de estratégias não compensatórias, eliminam por algum aspecto considerado relevante.

De acordo com Bettman, Luce e Payne (1998), as estratégias de decisão podem ser caracterizadas pelos aspectos do processamento da escolha. Para Kim, Chung e Paradice (1997), as estratégias de decisão podem ser classificadas em dois tipos: as estratégias compensatórias (ou decisão linear) e as não compensatórias (ou decisão não linear). A estratégia compensatória pressupõe que os respectivos valores à disposição em um determinado atributo são combinados de maneira aditiva, resultando em valor global. Essa estratégia permite a utilização de trade-offs entre um valor alto em uma dimensão e um valor baixo em outra dimensão (KIM; CHUNG; PARADICE, 1997; KAHN, 2005). Por outro lado, a estratégia não compensatória é indicada pelo uso não linear ou interativo de casos nos quais um valor baixo em uma dimensão não pode ser compensado por um valor alto em outra dimensão (WESTENBERG; KOELE, 1994).

Segundo Chu e Spires (2003), as estratégias compensatórias incluem a Adição de Pesos (WAD), a estratégia de Pesos Iguais (EQW) e a estratégia de Confirmação de Dimensões Majoritárias (MCD). Já as estratégias não compensatórias incluem a Lexicográfica (LEX), a Eliminação por Aspectos (EBA) e a estratégia Conjuntiva (CON) ou decisão satisfatória (ENGEL; BLACKWELL; MINIARD, 2000).

Conforme Bettman, Luce e Payne (1998), a estratégia de Adição de Pesos consiste em considerar uma alternativa num dado momento, examinar cada atributo para cada opção, 
Investigando o processo de escolha: uma análise das estratégias de decisão utilizadas na compra de televisores LCD

multiplicando o valor de cada atributo subjetivo ao seu peso e à sua importância, somando esses valores, adotando esse procedimento para todas as alternativas e escolhendo aquela de maior valor. Segundo Engel, Blackwell e Miniard (2000), sob essa estratégia, o indivíduo ocupa-se de julgamentos mais refinados sobre o desempenho das alternativas, além de aspectos de ser simplesmente favorável ou desfavorável.

A estratégia de Pesos Iguais consiste na análise de todas as alternativas e de todos os valores de atributos para cada alternativa. No entanto, essa estratégia simplifica a tomada de decisão por ignorar as informações sobre a importância relativa ou a probabilidade de cada atributo. Assim, um valor global de cada alternativa é obtido através da simples soma dos valores de cada atributo para essa alternativa (PAYNE; BETTMAN; JOHNSON, 1993), ou seja, o decisor simplesmente conta o número de vezes que cada alternativa é julgada favoravelmente, em termos do conjunto de critérios para avaliação, e a alternativa que obtiver o maior número de atributos positivos é a escolhida (ENGEL; BLACKWELL; MINIARD, 2000). Segundo Löbler (2005), a grande simplificação nessa estratégia reside no fato de não considerar uma ponderação dos pesos.

Na estratégia de Confirmação de Dimensões Majoritárias, as alternativas são processadas aos pares, nos quais os valores de duas alternativas são comparados em cada atributo e a alternativa majoritária é a vencedora. Por conseguinte, é realizado o mesmo processo de comparação par a par, até que todas as alternativas sejam avaliadas, restando uma única como vencedora (BETTMAN; LUCE; PAYNE, 1998).

A estratégia Lexicográfica determina o atributo mais importante e, em seguida, examina os valores de todas as alternativas relativas a esse atributo. Então, a alternativa com o melhor valor do atributo mais importante é selecionada (PAYNE; BETTMAN; JOHNSON, 1993). Se duas alternativas são percebidas como igualmente boas, elas são comparadas no segundo atributo mais importante. Esse processo continua até que o empate seja quebrado (ENGEL; BLACKWELL; MINIARD, 2000).

A estratégia de Eliminação por Aspecto, segundo Tversky (1972), consiste na seleção de um aspecto que elimine todas as alternativas que não incluem um valor mínimo no atributo mais importante, sendo que o processo continua até restar uma única alternativa. Se um determinado aspecto está incluído em todas as alternativas disponíveis, nenhuma é eliminada, e um novo aspecto é selecionado. Consequentemente, aspectos que são comuns a todas as alternativas consideradas não afetam as probabilidades de escolha (TVERSKY, 1972). Conforme o mesmo autor e ainda Engel, Blackwell e Miniard (2000), essa regra de 
decisão está intimamente relacionada com o modelo lexicográfico, no qual uma ordenação dos atributos relevantes é especificada a priori. Ainda, se nenhuma alternativa é selecionada, o indivíduo deve rever os cortes, usar uma estratégia de decisão diferente ou adiar a escolha (ENGEL; BLACKWELL; MINIARD, 2000).

A estratégia da decisão satisfatória é uma das mais antigas heurísticas identificadas no processo decisório (SIMON, 1955). Segundo Chu e Spires (2003), essa estratégia implica a definição de limiares para todos os atributos de uma só vez. A primeira alternativa que atender ou que exceder todos os limites dos valores será selecionada.

Baseado em Westenberg e Koele (1994), a divisão proposta por Harte, Westenberg e Someren (1994) agrupa a estratégia linear e a da diferença aditiva como compensatórias; e a eliminação por aspectos e a conjuntiva como não compensatórias. Confeccionando-se um resumo das estratégias decisórias, com uso da classificação proposta por Westenberg e Koele (1994), a configuração em dimensões e tipos fica como o exposto na Figura 1.

Harte, Westenberg e Someren (1994), baseados no processamento da informação, classificaram as estratégias de decisão em quatro tipos:

a) linear, na qual o decisor integra os aspectos de cada alternativa num valor total e escolhe a alternativa com o mais alto valor. Essa estratégia é similar à denominada adição de pesos de Bettman, Luce e Payne (1998);

b) conjuntiva, pela qual o decisor rejeita todas as alternativas com aspectos que não satisfazem um mínimo aceitável em um dos atributos, nesse caso, considerado o mais importante para aquele objeto. Este processamento é análogo ao tipo lexicográfico de Bettman, Luce e Payne (1998);

c) eliminação por aspectos, quando o decisor rejeita todas as alternativas com aspectos que não satisfazem um mínimo aceitável, inicia com o atributo mais relevante, seguido do segundo mais relevante e assim sucessivamente, escolhendo uma das alternativas remanescentes. Tal processamento apresenta a mesma denominação em Bettman, Luce e Payne (1998);

d) diferença aditiva, na qual o decisor avalia a diferença entre duas alternativas em cada atributo e soma cada uma delas, escolhendo a alternativa que tenha a mais ampla diferença positiva. Torna-se relevante salientar que este tipo não apresenta similar na classificação de Bettman, Luce e Payne (1998). 
Investigando o processo de escolha: uma análise das estratégias de decisão utilizadas na compra de televisores LCD

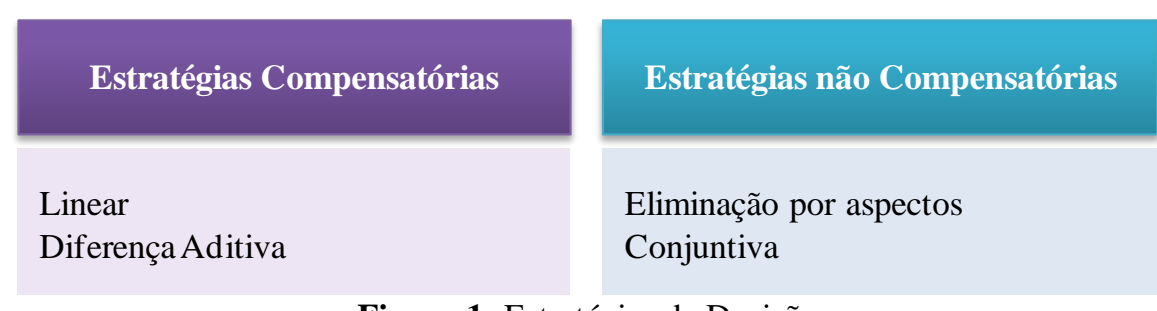

Figura 1- Estratégias de Decisão

Fonte: elaborado a partir de Westenberg e Koele (1994).

Nesse sentido, segundo Kim, Chung e Paradice (1997), os tomadores de decisão podem utilizar uma série de estratégias para isso, sendo diferentes uma das outras. Assim, os indivíduos, muitas vezes, acabam por utilizar estratégias de acordo com as informações ou com o conhecimento que possuem sobre o problema. De acordo com Löbler (2005), na fase inicial da decisão, indivíduos com menos conhecimento utilizam-se mais de uma estratégia não compensatória - eliminação por aspectos - do que indivíduos com mais conhecimento. Após essa primeira etapa, as estratégias passam a ser compensatórias para ambos os indivíduos, ou seja, com mais e com menos conhecimento, sem diferenças significativas.

\section{METODOLOGIA}

Visando-se a atender aos objetivos delineados neste estudo, realizou-se uma pesquisa de caráter qualitativo e de natureza exploratória, uma vez que, de acordo com Gil (1999), os estudos exploratórios proporcionam uma maior flexibilidade na busca de informações sobre determinado problema, visando a torná-lo mais claro e possibilitando a construção de novas ideias sobre o tema abordado.

Para o desenvolvimento deste estudo, utilizaram-se, como objeto de análise, televisores Liquid Crystal Display (LCD), visto que servem para responder à questão de pesquisa por apresentarem características bem definidas. Para verificação das estratégias de decisão do consumidor, realizaram-se entrevistas em profundidade, cujo roteiro foi elaborado com base em manuais técnicos e em questões abertas que permitiam ao entrevistado contribuir com o que julgasse relevante. Nessas entrevistas, com duração aproximada de 45 minutos, o processo de compra do aparelho televisor LCD foi descrito pelos dez entrevistados, selecionados por conveniência, considerando-se o fato de que haviam adquirido um televisor nos seis meses anteriores à entrevista.

As entrevistas foram gravadas, transcritas e organizadas de maneira que facilitassem a análise de acordo com a teoria apresentada. Para garantir a confidencialidade das informações e preservar a identidade dos entrevistados, optou-se por nomeá-los do A ao J. A 
partir da análise das entrevistas, foi possível identificar os atributos não considerados, os atributos eliminados e as compensações, ou trade offs, realizados durante o processo de decisão de compra dos entrevistados.

Cabe acrescentar que o modelo básico do processo decisório de Shimizu (2001) e as estratégias de decisão propostas por Westenberg e Koele (1994) na classificação de Harte, Westenberg e Someren (1994) foram a base para realização deste estudo. A Figura 2 sintetiza o modelo de pesquisa utilizado.

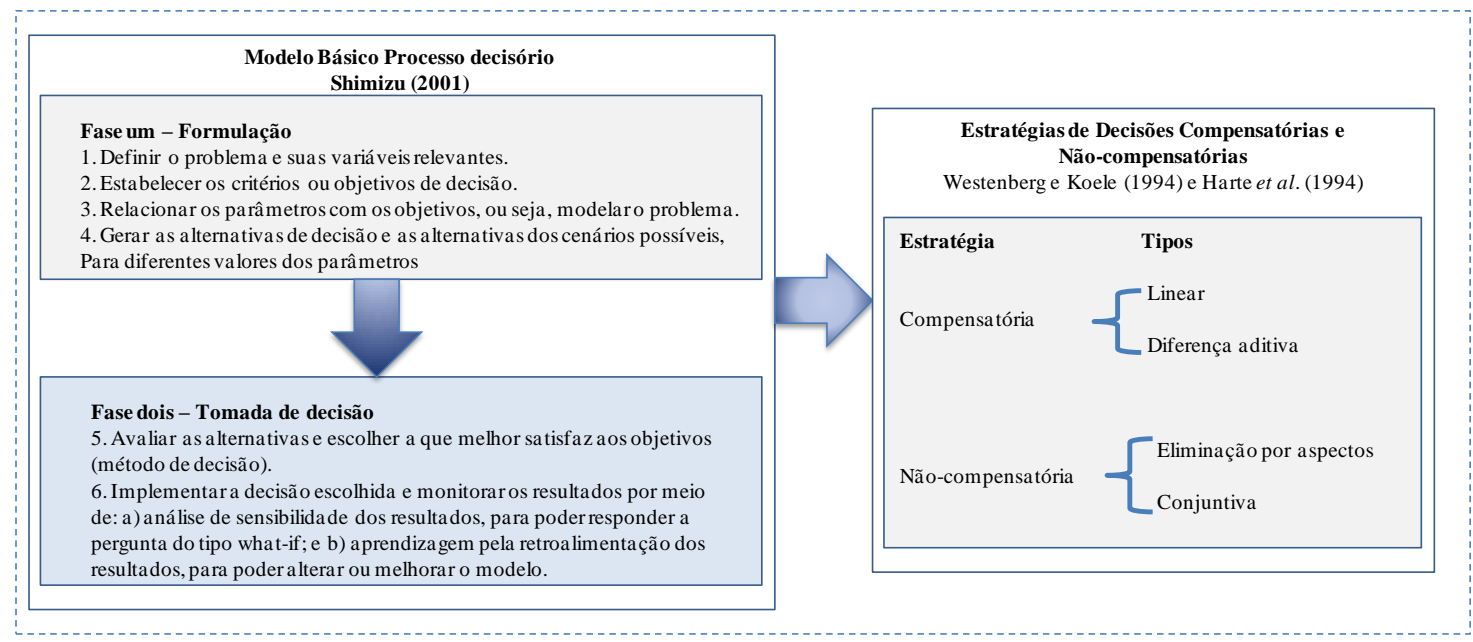

Figura 2- Modelo de Pesquisa

Fonte: elaborado pelos autores.

\section{ANÁLISE DOS RESULTADOS}

No que se refere ao perfil dos entrevistados, $50 \%$ pertencem ao sexo feminino e $50 \%$ ao masculino. A faixa etária predominante $(60 \%)$ é a dos 20 aos 30 anos, já com relação ao estado civil dos entrevistados, predominaram pessoas solteiras $(60 \%)$. Sobre a escolaridade da amostra geral investigada, percebe-se que $30 \%$ têm o terceiro grau completo e $30 \%$ o estão cursando.

\subsection{Tomada de decisão: as fases do processo}

Conforme modelo desenvolvido por Shimizu (2001), o processo decisório consiste em duas fases: a formulação do problema e a tomada de decisão. Na primeira fase, o decisor percebe e define a necessidade ou oportunidade de decisão, determinando os parâmetros relevantes ao processo. Neste estudo, observou-se que a primeira fase do processo de decisão dos entrevistados diz respeito à necessidade ou ao desejo de compra de um televisor LCD, conforme pode ser evidenciado nas seguintes falas: 
Investigando o processo de escolha: uma análise das estratégias de decisão utilizadas na compra de televisores LCD

porque eu não tinha televisão na sala e porque eu queria uma televisão maior (Entrevistado D).

O primordial para a compra da TV foi o play 3 e depois os filmes que eu estou baixando em qualidade superior, agora eu estou baixando tudo em 1080, fica perfeito, ainda mais em blu-ray, fica perfeita a imagem (Entrevistado C).

Ainda na primeira fase do processo decisório, os indivíduos estabelecem alguns parâmetros a respeito do produto, relacionados à necessidade ou ao desejo de compra (SHIMIZU, 2001). Assim, o consumidor constrói alternativas de compra de produtos que, nesse caso, podem ser definidas pelos modelos de televisores LCD, como observado na fala do entrevistado C: "essa TV tem entrada USB, que dá para ver filme direto no pen-drive, filme, foto, tudo, e a velocidade de transmissão de dados que é $600 \mathrm{~Hz}$ ”.

A segunda fase do processo decisório refere-se à tomada de decisão propriamente dita, na qual o decisor avalia as alternativas geradas a partir dos parâmetros definidos na fase um e escolhe a que melhor satisfaz seus objetivos (SHIMIZU, 2001). Neste estudo, percebeuse que o decisor desempenhou o seu processo de escolha similar ao modelo proposto por Shimizu (2001), relacionando as alternativas de produto com o seu desejo ou a sua necessidade, definidos anteriormente.

Quando o indivíduo, após avaliar as alternativas com relação aos atributos e estar convicto que suprem a necessidade percebida, faz uso das estratégias de decisão, ele dá início efetivamente ao processo de tomada de decisão. O início do uso de estratégias de decisão ocorre após a formulação do problema, coincidindo com a fase dois do modelo proposto por Shimizu (2001). Segundo Westenberg e Koele (1994), as estratégias de decisão são avaliações do processo que conduz a uma escolha, baseadas no modo como os indivíduos processam as informações disponíveis, considerando alternativas com múltiplos atributos, a fim de chegar a uma escolha final. Ainda, como última ação do processo, é realizado um monitoramento dos resultados (SHIMIZU, 2001), o que pode ser observado na fala do entrevistado D:

eu sempre comprei aparelho DVD, som, home teather, eu sempre comprei [...] ou [...], não sei afirmar tecnicamente se é a melhor, mas eu sempre comprei essa marca. Eu comprei um home teather junto, então preferi uma marca que fosse as duas marcas iguais, para que fossem mais compativeis, as duas são [...], eu levei em conta o home junto e a TV.

Quanto ao uso das estratégias de decisão, inicialmente os decisores utilizaram as estratégias não compensatórias, eliminando algumas alternativas do processo. As estratégias não compensatórias são aquelas nas quais o tomador de decisão não busca compensar valores,

REAd | Porto Alegre - Edição 77 - N 1 - janeiro/abril 2014 - p. 170-194 
ou seja, um valor baixo em uma dimensão não pode ser compensado por um valor alto em outra dimensão (WESTENBERG; KOELE, 1994; ENGEL, BLACKWELL; MINIARD, 2000). Logo, as alternativas que não têm relevância no processo de escolha do decisor são eliminadas. Um exemplo disso pode ser verificado na seguinte fala:

Eu decidi comprar uma TV de 32, então, já eliminei as de outro tamanho, assim como exclui da lista aquelas que não tinham entrada HDMI, isso eu considerei. Imagem e som, tudo considerei (Entrevistado D).

Num segundo momento, os decisores, frente a um número menor de alternativas, passam a fazer trade-offs, ou trocas, situações, nas quais um valor baixo em uma dimensão pode ser compensado por um valor alto em outra dimensão, fazendo-se uso de estratégias compensatórias (KIM; CHUNG; PARADICE, 1997; ENGEL; BLACKWELL; MINIARD, 2000; KAHN, 2005). O uso de estratégias compensatórias presume que os respectivos valores à disposição em um determinado atributo são combinados de maneira aditiva, resultando em valor global. A fala do entrevistado $\mathrm{G}$ representa esse contexto:

O televisor $X$, na loja $W$ estava $R \$ 1.990,00$. O mais barato que tinha na Internet era $R \$ 2.990,00$, então, era uma excelente promoção de preço, tanto que eu queria uma $Y$ e comprei a $X$, foram $R \$ 1.000$ reais de diferença pra uma $X$ de mesma polegada, aí a gente optou pela X de 40", não de 42", porque não tem de 42", mas por 2 " $R \$ 1.000$ reais de diferença, eu achei que valia a pena. (Entrevistado G).

Ampliando-se a análise sobre o uso das estratégias de decisão, observou-se que os atributos considerados pelos consumidores para eliminação das alternativas foram: tamanho, conexões pré-requeridas, marcas desconhecidas, recursos de áudio e imagem, Picture in Picture (PIP), vida útil, faixa de preço, design e ajustes. Com as eliminações de produtos que não satisfizeram o mínimo aceitável em algum atributo, restaram alternativas para a realização de trade-offs, ou seja, a compensação entre atributos dos produtos, conforme demonstra a fala do entrevistado C: "é, eu já meio que eliminei as TVs que não tinham entrada USB, porque essa é uma grande vantagem, eu acho”.

Assim, o uso de estratégias compensatórias ocorreu entre os produtos que continham características definidas como determinantes para escolha e compra. As compensações feitas aconteceram entre os seguintes atributos: preço, marca, forma de pagamento, tamanho, recursos de imagem, conexões e vida útil. Dessa forma, as decisões se caracterizam como ótimas, a exemplo das falas dos seguintes entrevistados:

Os critérios levados em consideração foram preço, principalmente, vamos dizer assim, e também considerei a marca. (Entrevistado G).

REAd | Porto Alegre - Edição 77 - N 1 - janeiro/abril 2014 - p. 170-194 
Investigando o processo de escolha: uma análise das estratégias de decisão utilizadas na compra de televisores LCD

A taxa de contraste, que é muito maior nessa daqui, ela tem entrada USB, que dá para ver filme direto no pen-drive. (Entrevistado C).

Dada a exposição dos resultados, é possível perceber que os atributos design, ajustes, forma de pagamento e PIP foram os que não coincidiram no uso de ambas as estratégias de decisão. Isso equivale a dizer que os demais atributos acima mencionados fizeram-se representar tanto no uso de estratégias não compensatórias quanto no uso de estratégias compensatórias.

\subsection{Características que diferenciam as estratégias de decisão}

Através da análise das entrevistas, observou-se que a característica individual que diferencia o uso de estratégias de decisão dos consumidores, ou seja, o uso de estratégias não compensatórias para estratégias compensatórias é o conhecimento prévio do produto. Segundo Mora (2001), conhecimento é definido como a apreensão de um objeto por um sujeito, isso equivale a dizer que o sujeito o representa e, quando representa o objeto tal qual é, o sujeito tem um conhecimento verdadeiro do mesmo (MORA, 2001).

Neste estudo, pode-se dizer que os consumidores C, D, F, G, H e J, que, antes da escolha final de compra, buscaram informações a respeito do produto, tais como desempenho, recursos e possíveis conexões, foram considerados como consumidores com conhecimento do produto. Cabe salientar que a busca do conhecimento acontece durante o processo de decisão de compra, após o indivíduo ter identificado a necessidade a ser satisfeita e a relacionado com as alternativas existentes.

Depois de ter passado por essa fase do processo de decisão, alguns indivíduos perceberam uma lacuna entre o conhecimento que possuíam acerca do produto e o necessário para realizar a melhor escolha. Por esse motivo, os entrevistados buscaram as informações sobre o produto em fóruns, contato com técnicos, manuais do produto, experiências passadas relacionadas ao atributo marca, dentre outros, o que pode ser observado na seguinte fala:

Levei dois anos para comprar a TV, na verdade, antes de comprar eu resolvi aprender sobre o que eu estou comparando, não simplesmente [...], ah eu vou comprar uma TV, eu não compro assim, eu cheguei a ler quase todos os manuais, das séries dos modelos que vi, baixei da Internet o manual e li o manual, eu não li as especificações do produto. Depois que eu comecei a entender a linguagem do produto, entendi, ler, aprender o que é o HD, o full HD, o que é contraste, esse brilho, qual é a diferença de uma TV Plasma, de uma LCD, para uma Led, saber o que o produto oferece. (Entrevistado $\mathrm{H}$ ).

entrei nos fóruns, para ver o que o pessoal dizia e o preço que era mais baixo do que as outras. (Entrevistado C).

REAd | Porto Alegre - Edição 77 - N 1 - janeiro/abril 2014 - p. 170-194 
Eu tenho um amigo que faz faculdade de engenharia mecatrônica, eu pedi para ele qual que ele achava, e ele me ajudou a pesquisar. (Entrevistado D).

procurei conhecer todas as conexões possíveis. Conheço todas e levei todas em consideração. (Entrevistado J).

Por outro lado, foram considerados consumidores com pouco conhecimento aqueles que não tiveram a iniciativa de buscar informações e aprender sobre os atributos do produto. Nesta pesquisa, os entrevistados A, B, E e I foram considerados como consumidores com pouco conhecimento, conforme fala do sujeito I:

Não tive preocupações técnicas muito grandes, levei em consideração o tamanho
que eu queria [...] eu tomei uma decisão considerando o tamanho da tela que eu
queria e daí eu fui perseguindo essa ideia, eu queria comprar a [...], daí eu olhei as
duas ligadas e comparei as imagens (Entrevistado I).

Através da análise das entrevistas, observou-se que, durante o processo de escolha do televisor LCD, os consumidores com pouco conhecimento a respeito do produto utilizaram, em maior número, as estratégias não compensatórias. Com isso, tais consumidores eliminaram o produto que não satisfez o mínimo aceitável em algum atributo.

Cabe acrescentar que, tanto para os consumidores com conhecimento sobre o produto como para os consumidores com pouco conhecimento, a etapa de identificação dos desejos e das necessidades da compra do televisor acontece da mesma forma. No entanto, a definição dos atributos e a relação com os parâmetros aconteceram de forma diferenciada, pois os consumidores com conhecimento estabeleceram atributos mais específicos, levando em consideração o desempenho e características de performance do produto, conforme pode ser observado nas seguintes falas:

[...] que apresentasse recursos que hoje uma TV comum não tem, eu posso ligar DVD, câmera, computador, câmera digital, eu posso ligar um USB, fotografias, colocar no pen-drive e ligar direto nela. (Entrevistado $\mathrm{H})$.

[...] procurei todas as conexões possíveis. Conheço todas e levei todas em consideração. (Entrevistado J).

A falta de conhecimento do número total de recursos do produto e os benefícios por ele proporcionados provocam eliminação das alternativas através de características que não levam em consideração o desempenho do produto, uma vez que não foram ponderados recursos específicos. Dessa forma, as decisões nem sempre são ótimas, mas sim satisfatórias naquela situação e naquele momento (SIMON, 1955), conforme se pode observar na fala do entrevistado A:

REAd | Porto Alegre - Edição 77 - N 1 - janeiro/abril 2014 - p. 170-194 
Investigando o processo de escolha: uma análise das estratégias de decisão utilizadas na compra de televisores LCD

Eu queria um televisor para assistir no meu quarto, que não fosse pequeno, nem grande demais, que tivesse conexão para videogame e DVD, que fosse de boa qualidade, que tivesse assistência na minha cidade, que tivesse preço abaixo de $R \$$ 600,00, que eu pudesse pagá-lo à vista e que me dessem desconto por isso.

A análise dos atributos utilizados na etapa não compensatória para a eliminação de alternativas e na etapa compensatória para tomar a melhor decisão de acordo com o estabelecido na fase um, permite que se infira que as decisões de consumidores com menos conhecimento não são decisões ótimas, apenas satisfatórias em dada situação e em dado momento (SIMON, 1955).

\subsection{Identificando-se o início dos trade-offs}

O início do uso de trade-offs marca a mudança de estratégia de decisão. Segundo Payne, Bettman e Johnson (1993), essa mudança ocorre quando as pessoas se deparam com problemas de decisão que envolvem duas ou três alternativas.

A partir da compilação dos resultados, observou-se que a troca de estratégia não compensatória para estratégia compensatória aconteceu no momento em que restavam dois ou três televisores LCD para escolha. Dessa forma, salienta-se que os tomadores de decisão não se utilizaram dos mesmos atributos na etapa compensatória, exceto em relação ao atributo preço, identificado como atributo compensado em todos os processos, conforme ilustrado na Figura 3. 
Mauri Leodir Löbler, Eliete dos Reis \& Larissa Medianeira Bolzan

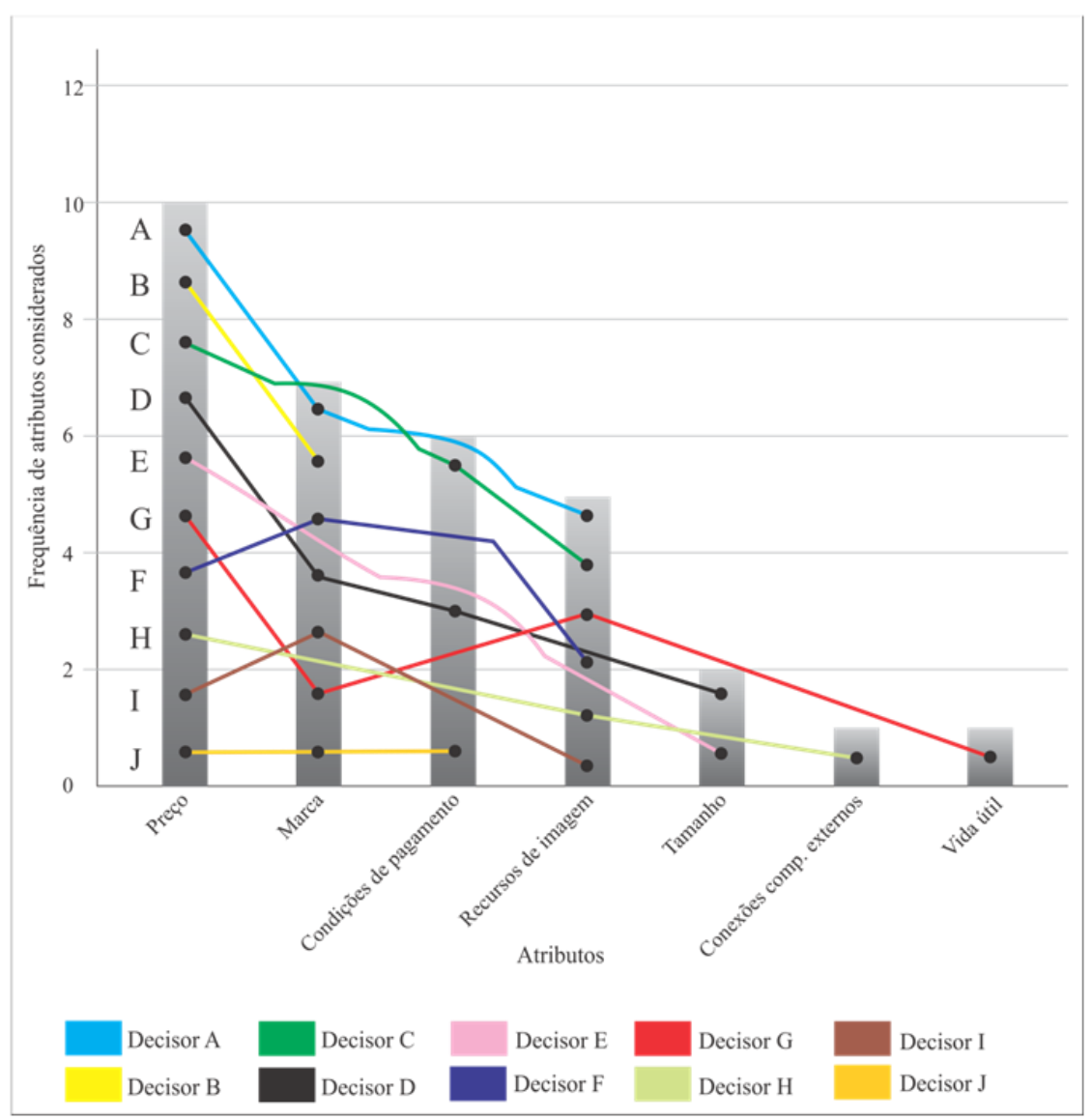

Figura 3- Compensações de atributos

Fonte: Dados da pesquisa.

A partir da análise da Figura 3, podem-se observar os trade-offs realizados pelos decisores por meio da estratégia compensatória linear, na qual o decisor integra os aspectos de cada alternativa num valor total e escolhe a alternativa com o mais alto valor (HARTE, WESTENBERG e SOMEREN 1994; ENGEL; BLACKWELL; MINIARD, 2000). As linhas da figura representam o indivíduo, e a combinação dos pontos representam os atributos em que os mesmos realizaram os trade-offs.

Os decisores $\mathrm{G}$ e $\mathrm{D}$ foram os que realizaram o maior número de compensações. $\mathrm{O}$ decisor D compensou os atributos preço, marca, condições de pagamento e tamanho; já o decisor $\mathrm{G}$ compensou preço, marca, recursos de imagem e vida útil. Pode-se notar que os atributos preço e marca foram uniformes a ambos os decisores. Os decisores B e E foram os que realizaram o menor número de compensações, fazendo trade-offs apenas entre preço e marca (decisor B) e preço e tamanho (decisor E). Nota-se também, com base na Figura 3, que, ao contrário do atributo preço, que foi o mais considerado na realização de trade-offs, atributos como conexões e vida útil foram os menos ponderados. 
Investigando o processo de escolha: uma análise das estratégias de decisão utilizadas na compra de televisores LCD

\subsection{Uso de estratégias de decisão}

O uso de estratégias de decisão pelos consumidores participantes da pesquisa, independente do conhecimento que tinham acerca do produto, ocorreu da seguinte forma: num primeiro momento, os indivíduos utilizaram a estratégia não compensatória, especificamente a de eliminação por aspectos. Segundo Harte, Westenberg e Someren (1994) e Engel, Blackwell e Miniard (2000), a estratégia de eliminação por aspectos ocorre quando o decisor rejeita todas as alternativas com aspectos que não satisfazem um mínimo aceitável. Tal processo inicia com o atributo mais relevante, seguido do segundo mais relevante e assim sucessivamente, escolhendo uma das alternativas remanescentes.

Para os consumidores com conhecimento, os atributos eliminados através da estratégia não compensatória, eliminação por aspectos, a fim de que reduzissem o número de alternativas, foram: tamanho do televisor, ajustes desejados, recursos de imagem, PIP, conexões e marcas desconhecidas. Já os consumidores com pouco conhecimento eliminaram os atributos: tamanho do televisor, vida útil, design, preço e conexões.

Num segundo momento, após o uso da estratégia não compensatória (eliminação por aspecto), o decisor com conhecimento acerca do produto utilizou-se de estratégias compensatórias, especificamente a linear. Segundo Harte, Westenberg e Someren (1994), na estratégia linear, os indivíduos atribuem pesos aos atributos a serem compensados, avaliam cada um deles nas alternativas restantes, integram os valores dos atributos num valor total em cada alternativa e escolhem a alternativa com mais alto valor.

No que se refere à estratégia de decisão compensatória, os atributos utilizados através da estratégia linear pelos consumidores com conhecimento, para realizações de tradeoffs (trocas) foram: preço, marca, forma de pagamento, tamanho, recursos de imagem, conexões e vida útil. Quanto aos consumidores com pouco conhecimento, os atributos foram: preço, marca, forma de pagamento, tamanho e recursos de imagem. O Quadro 1 ilustra a comparação entre os atributos utilizados na fase dois do processo de escolha do televisor, na qual foram utilizadas as estratégias de decisão não compensatórias e compensatórias.

Quadro 1 - Estratégias de decisão utilizadas no processo de escolha

\begin{tabular}{|c|c|c|}
\hline $\begin{array}{c}\text { Atributos em que foram } \\
\text { utilizadas }\end{array}$ & Decisores com conhecimento & $\begin{array}{l}\text { Decisores com pouco } \\
\text { conhecimento }\end{array}$ \\
\hline $\begin{array}{l}\text { Estratégias Não Compensatórias } \\
\text { (Eliminação por aspecto) }\end{array}$ & $\begin{array}{l}\text { PIP } \\
\text { Conexões } \\
\text { Faixa de tamanho } \\
\text { Ajustes } \\
\text { Marcas desconhecidas } \\
\text { Recursos de imagem }\end{array}$ & $\begin{array}{l}\text { Faixa de tamanho } \\
\text { Faixa de preço } \\
\text { Vida útil } \\
\text { Conexões } \\
\text { Design }\end{array}$ \\
\hline
\end{tabular}

REAd | Porto Alegre - Edição 77 - N 1 - janeiro/abril 2014 - p. 170-194 
Mauri Leodir Löbler, Eliete dos Reis \& Larissa Medianeira Bolzan

\begin{tabular}{|c|l|l|}
\hline \multirow{2}{*}{\begin{tabular}{|l|l|} 
Estratégias Compensatórias \\
(Linear)
\end{tabular}} & Preço & Preço \\
& Forma de pagamento & Marca \\
& Recursos de imagem & Tamanho \\
& Conexões & Recursos de imagem \\
& Vida útil & Tamanho \\
\hline
\end{tabular}

Fonte: elaborado pelos autores.

Os atributos não considerados na decisão de compra do televisor que coincidiram para ambos os consumidores, com conhecimento e com pouco conhecimento, foram: ângulo de visualização, atendimento, assistência técnica, experiências passadas, garantia, HDMI, idioma, influências, loja, publicidade, recursos analógicos, sinal digital e tempo de resposta. Acrescenta-se, para os consumidores com conhecimento, os atributos design e a faixa de preço, e para consumidores com pouco conhecimento, os atributos PIP, os ajustes e as marcas desconhecidas. A Figura 4 apresenta os atributos que não foram considerados pelos consumidores.

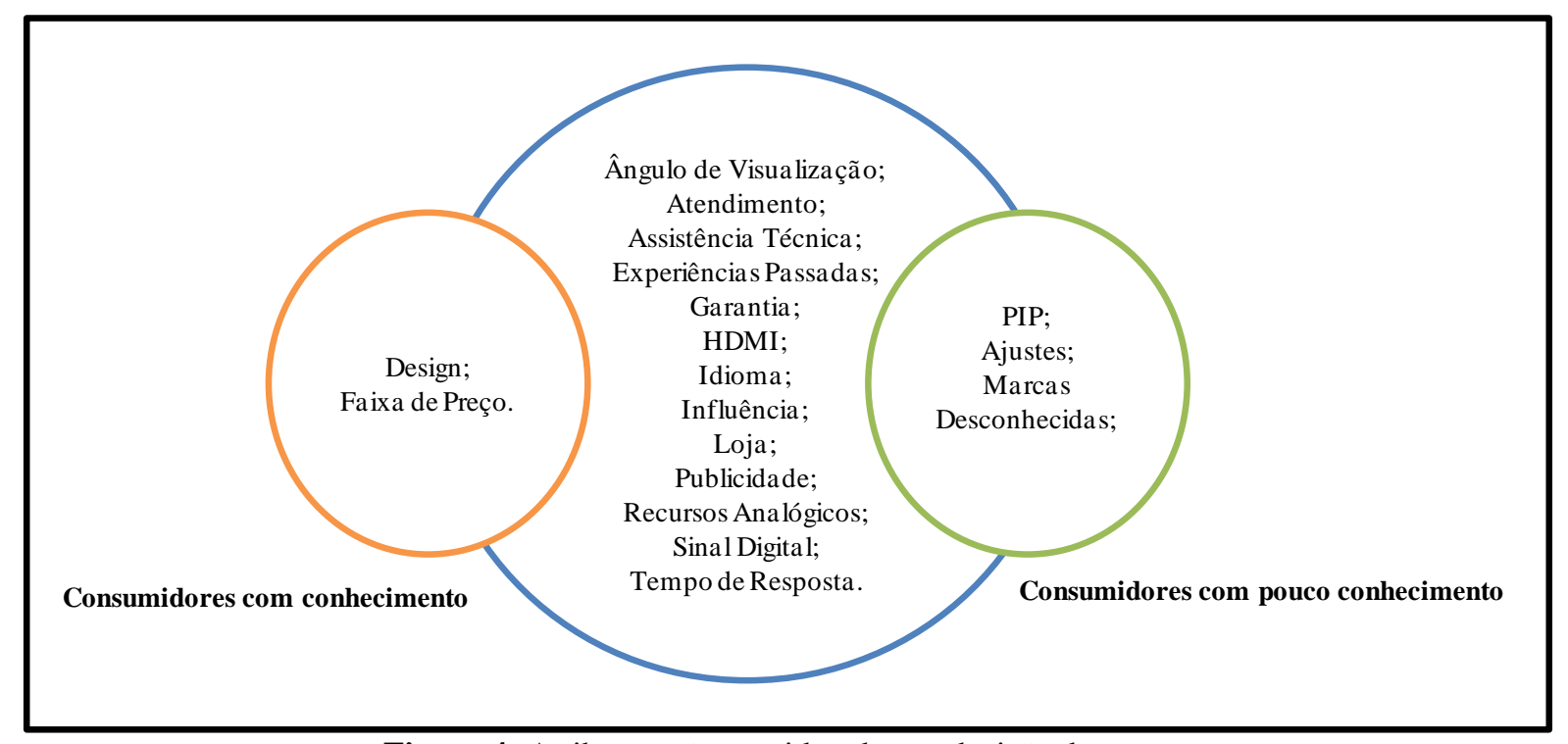

Figura 4- Atributos não considerados na decisão de compra

Fonte: Dados da pesquisa

A análise das entrevistas permitiu constatar que os consumidores com conhecimento acerca do produto utilizam-se mais de estratégias compensatórias e, no processo final de decisão, acabam por compensarem maior número de atributos para decidirem entre uma alternativa, tomando, assim, uma decisão ótima. Por outro lado, os consumidores com pouco conhecimento, mas que também utilizam estratégias compensatórias, no final do processo decisório, fazem-no considerando um menor número de atributos.

Consumidor com conhecimento a respeito do produto, entrevistado $\mathrm{C}$ :

REAd | Porto Alegre - Edição 77 - N 1 - janeiro/abril 2014 - p. 170-194 
Investigando o processo de escolha: uma análise das estratégias de decisão utilizadas na compra de televisores LCD

Levei em consideração vida útil, taxa de contraste, que é muito maior nessa tv aqui, ela tem entrada USB, que dá para ver filme direto no pen-drive, e ainda mais em blu-ray, conexão para o play 3, fica perfeita a imagem, a velocidade de transmissão de dados que é de 600hz. Daí eu fiz comparação com o custo, né, o preço dessa era mais baixo do que as outras. Mas foi primordial a entrada pra o play 3, a qualidade de imagem e o preço. (Entrevistado C).

Consumidor com pouco conhecimento a respeito do produto, entrevistado E:

Eu pensei 32" pra cozinha vai ser ideal, não precisa ser maior, porque eu não tinha tv pra cozinha. [...] E, assim, em relação à marca, o que me ponderou foi, assim, ela é uma tv relativamente com a marca nova, em termos de televisor, mas monitores, da mesma marca, já tem há anos no mercado, e eu nunca vi dá problemas em relação a monitores, né, da marca, então, eu olhei, vi pelo preço, a questão do tamanho, era um tamanho, pra mim, ideal e a marca né, acreditei eu, no momento, que seria uma marca boa por causa da sua fama em relação a monitores, né. (Entrevistado E).

Dessa forma, pode-se dizer que as fases do processo de tomada de decisão, bem como as estratégias de decisão utilizadas, tanto para consumidores com conhecimento como para consumidores com pouco conhecimento, são as mesmas.

\section{CONSIDERAÇÕES FINAIS}

Diferentes estratégias são utilizadas pelos indivíduos para tomar decisões. Dessa forma, o decisor pode realizar ou implementar o processo de escolha de diferentes formas (BETTMAN; ZINS, 1977), o que sugere certa flexibilidade de resposta diante da ampla variedade de condições de tarefa.

Nesse contexto, a presente pesquisa teve como objetivo analisar a forma como os indivíduos se utilizam de estratégias compensatórias e não compensatórias nas decisões de compra. Além desse objetivo, o estudo apresentou objetivos secundários, como: (i) identificar características nos indivíduos pesquisados que possam diferenciar estratégias de decisão de compra; e (ii) verificar em que ponto os indivíduos mudam o tipo de estratégia de decisão.

Os resultados da pesquisa permitiram observar que, independente do conhecimento acerca do problema, o processo decisório ocorre sob duas fases: a primeira, em que é percebido o desejo ou a necessidade de possuir o produto, relacionando parâmetros e gerando alternativas, conforme Shimizu (2001); a segunda fase, em que o consumidor utiliza estratégias de decisão, caracterizadas como não compensatórias - eliminação por aspectos e compensatórias - linear para escolha do produto.

REAd | Porto Alegre - Edição 77 - N 1 - janeiro/abril 2014 - p. 170-194 
Com relação à característica individual que diferencia as estratégias de decisão de compra dos consumidores, os dados da pesquisa mostraram que o conhecimento prévio do produto é o que determina a utilização de diferentes estratégias de decisão. Os decisores que, antes do processo final de compra, buscaram informações a respeito do produto, tais como desempenho, recursos e possíveis conexões, foram caracterizados como consumidores com conhecimento do produto. Os consumidores com pouco conhecimento foram os que não tiveram a iniciativa de buscar informações e aprender sobre os atributos do produto.

Nesse contexto, diferentes estratégias de decisão foram utilizadas pelos indivíduos, algumas envolvendo o processamento de todas as informações com relação às alternativas disponíveis, expressando trade-offs, ou trocas; outras envolvendo estratégias de simplificação (heurística), usando informações mais selecionadas e, consequentemente, evitando essas trocas.

De acordo com Payne, Bettmann e Johnson (1993), quando as pessoas se deparam com problemas de decisão que envolvem exatamente duas ou três alternativas, frequentemente utilizam estratégias de decisão que processam todas as informações relevantes e determinam explicitamente uma, para decidir em que medida estão propensos a fazer tradeoff, ou trocas, de um atributo menos valorizado para outro atributo mais valorizado. Nesse sentido, o ponto em que os indivíduos mudaram o tipo de estratégia de decisão coincidiu com quando os decisores, frente a duas ou três alternativas para a escolha final, iniciaram os tradeoffs, caracterizando, assim, a mudança de estratégia de decisão de não compensatória para compensatória.

Assim, conclui-se que, na etapa não compensatória do processo de escolhas, os tomadores de decisão, tanto aqueles com conhecimento a respeito do objeto de decisão quanto os com pouco conhecimento, eliminaram todas as alternativas cujos atributos não satisfizeram o nível aceitável, essa estratégia é classificada por Harte, Westenberg e Someren (1994) como eliminação por aspectos. Na etapa compensatória, os tomadores de decisão, também indiferentes quanto ao nível de conhecimento a respeito do produto, atribuem pesos aos atributos a serem compensados, avaliam cada um deles nas alternativas restantes, integram os valores dos atributos num valor total, em cada alternativa, e escolhem a alternativa com mais alto valor, essa estratégia é classificada por Harte, Westenberg e Someren (1994) como linear.

Salienta-se que os tomadores de decisão não se utilizaram dos mesmos atributos na etapa compensatória, uma vez que, nessa etapa, foi identificada maior diferença entre as escolhas dos consumidores com diferentes níveis de conhecimento. Isso se deu porque mais 
Investigando o processo de escolha: uma análise das estratégias de decisão utilizadas na compra de televisores LCD

atributos foram considerados pelos consumidores com maior conhecimento, a respeito do produto, além de as respectivas características levarem em conta o desempenho do televisor, ou seja, a sua performance frente aos outros. A Figura 5 ilustra a síntese dos resultados da pesquisa.

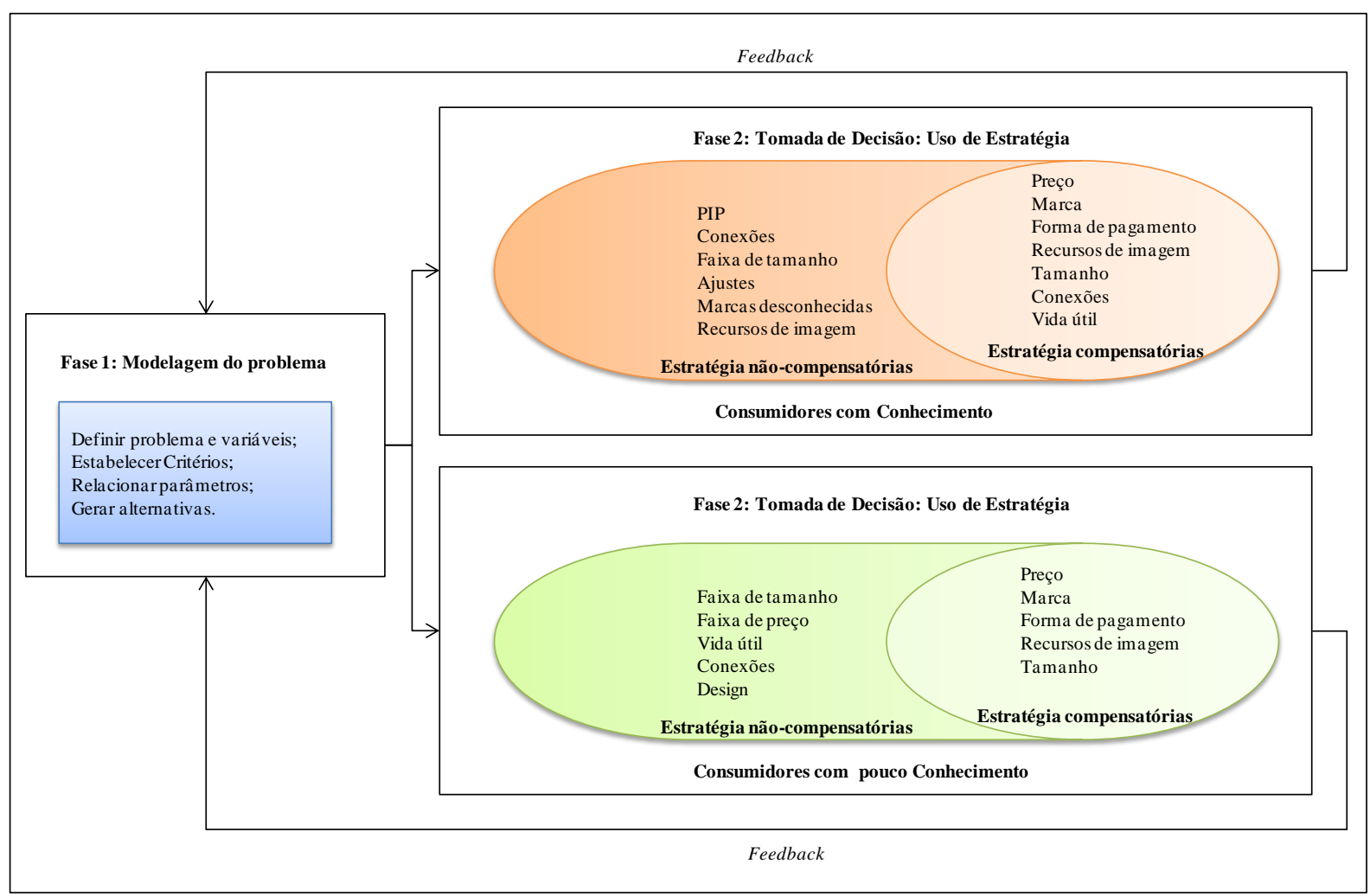

Figura 5- Síntese dos resultados

Fonte: elaborado pelos autores.

Para o campo especifico de estudo sobre processo decisório, este trabalho torna-se relevante por permitir a compreensão de como os indivíduos tomam decisões frente a uma diversidade de alternativas. Como limitações do estudo, pode-se citar a falta de acompanhamento durante o processo de escolha do televisor LCD, por parte dos consumidores, pois as entrevistas foram após a compra do produto. Considera-se também uma limitação os dados coletados para a amostra, já que foram apenas dez entrevistados. Sugerese, para pesquisas futuras, a realização de estudos sobre essa temática, que combinem abordagens qualitativas e quantitativas, abrindo espaço para pesquisas complementares, descritivas e explicativas. Quanto à realização de novos estudos sobre estratégias de decisão, acrescenta-se a sugestão de acompanhamento por parte do pesquisador durante o processo de escolha do consumidor. 
Mauri Leodir Löbler, Eliete dos Reis \& Larissa Medianeira Bolzan

\section{REFERÊNCIAS}

BETTMAN, J. R.; LUCE, M. F.; PAYNE, J. W. Constructive consumer choice processes. In: Journal of Consumer Research, v.25, p. 187-217, dez. 1998.

BETTMAN, J. R.; ZINS, M. A. Constructive processes in consumer choice. Journal of Consumer Research, v. 4, set. 1977.

BROOKS, L.; LEBLANC, V. R.; NORMAN, G. R. On the difficulty of noticing obvious features in patient appearance. Psychonomic Bulletin and Review, v.11, p. 112-117, 2000.

BUENO, L. A.; YAMAGUTI, C. L. Decisões organizacionais e de consumo: Um modelo genérico. Artigos e Ensaios, v. 4, n. 12, 2009.

CHU, P. C.; SPIRES, E. E. Persceptions of accurancy and effort of decision strategies. Organizational Behavior and Human Decision Processes, v. 91, p. 202-214, 2003.

DIECKMANN, A.; DIPPOLD, K.; DIETRIECH, H. Compensatory versus non-compensatory models for predicting consumer preferences. Judgment and Decision Making, v. 4, n. 3 p. 200-213, 2009.

ENGEL, J. F.; BLACKWELL, R. D.; MINIARD, P. W. Comportamento do consumidor. 8. ed. Rio de Janeiro: LTC, 2000.

GIL, A. C. Métodos e técnicas de pesquisa social. São Paulo: Atlas, 1999.

HARRISON, E. F. A process perspective on strategic decision making. Management Decision, v. 34, p. 46-53, 1996.

HARTE, J. M.; WESTENBERG, M. R. M.; SOMEREN, M. V. Process models of decision making. In: Acta Psychologica, v. 87, 1994.

KAHN, B. E. The Power and limitations of social relational framing for understanding consumer decision processes. Journal of Consumer Psychology, v. 15, p. 28-34, 2005.

KIM, C. N.; CHUNG, H. M.; PARADICE, D. B. Inductive modeling of expert decision making in loan evaluation: a decision strategy perspective. Decision Support System, v. 21, p. 83-98, 1997.

KIM, C. N.; YANG, K. H.; KIM, J. Human decision-making behavior and modeling effects. Decision Support System, v. 45, p. 517-527, 2008. 
Investigando o processo de escolha: uma análise das estratégias de decisão utilizadas na compra de televisores LCD

LESGOLD, A.; RUBINSON, H.; FELTOVICH, P.; GLASER, R.; KLOPFER, D.; WANG, Y. Expertise in a complete skill: diagnosing x-ray pictures. In: CHI, M. T. H.; GLASER, R.; FARR, M. J. The nature of expertise. NJ.: Lawrence Erlbaum Associates, p. 310-342, 1988.

LÖBLER, M. L. Processamento da informação: uma avaliação dos diferentes níveis de conhecimento no processo de decisão. 2005. 215f. Tese (Doutorado em Administração)Universidade Federal do Rio Grande do Sul, Porto Alegre, 2005.

LÖBLER, M. L.; ESTIVALETE, V. F. B. Análise da decisão através do uso da informação e das estratégias de decisão interagindo com um SAD. In: SEMEAD, 11., 2008, São Paulo. Anais... São Paulo, 2008.

MARKMAN, A. B.; MEDIN, D. L. Decision making. In: MEDIN, D. L.; PASHLER, H. (Ed.). Stevens handbook of experimental psychology. New York: John Wiley and Sons, 2001. p. 413-466.

MORA, J. F. Dicionário de filosofia. São Paulo: Martins Fontes, 2001.

PAYNE, J. W.; BETTMAN, J. R.; JOHNSON, E. J. The adaptive decision maker. Cambridge University Press, 1993.

SHIMIZU, T. Decisão nas organizações: introdução aos problemas de decisão encontrados nas organizações e nos sistemas de apoio a decisão. São Paulo: Atlas, 2001.

SIMON, H. A. A behavioral model of rational choice. Quarterly Journal of Economics, v.69, p. $99-118,1955$.

TODD, P.; BENBASAT, I. An experimental investigation of the impact of computer based decision aids on decision making strategies. Decision Support System, p. 87-115, jul. 1991.

TVERSKY, A. Elimination by aspects: a theory of choice. Psychological Review, v. 79, n. 4, p. 281-299, 1972.

WESTENBERG, M. R. M.; KOELE, P. Multi-attribute evaluation process: methodological and conceptual issues. Acta Psychologica, v. 87, p. 65-84, 1994. 